CLINICAL STUDY

\title{
Association between menstrual cycle irregularities and endocrine and metabolic characteristics of the polycystic ovary syndrome
}

Dimitrios Panidis, Konstantinos Tziomalos ${ }^{1}$, Panagiotis Chatzis, Efstathios Papadakis, Dimitrios Delkos, Elena A Tsourdi, Eleni A Kandaraki and Ilias Katsikis

Division of Endocrinology and Human Reproduction, Second Department of Obstetrics and Gynecology, Hippokration Hospital, Aristotle University of Thessaloniki, Thessaloniki, Greece and ${ }^{1}$ First Propedeutic Department of Internal Medicine, AHEPA Hospital, Aristotle University of Thessaloniki, 1 Stilponos Kyriakidi Street, 54636 Thessaloniki, Greece

(Correspondence should be addressed to K Tziomalos; Email: ktziomalos@yahoo.com)

\begin{abstract}
Objective: Insulin resistance (IR) is frequent in polycystic ovary syndrome (PCOS) and contributes to the increased risk for type 2 diabetes mellitus and cardiovascular disease of this population. Several markers of IR are used but most are expensive or have limited sensitivity and specificity. Preliminary data suggest that the menstrual cycle pattern correlates with IR in PCOS but existing studies are small. We aimed to assess the relationship between the type of menstrual cycle irregularities and IR in PCOS. Design: Prospective study.

Methods: We studied 1285 women with PCOS, divided according to the menstrual cycle pattern.

Results: Patients with isolated secondary amenorrhea and those with secondary amenorrhea alternating with regular menstrual cycles were more insulin resistant than patients with regular cycles (Group D). Patients with isolated oligomenorrhea were also more insulin resistant than Group D. However, patients with oligomenorrhea alternating with regular cycles, secondary amenorrhea, or polymenorrhea had comparable levels of markers of IR with Group D. Moreover, patients with oligomenorrhea alternating with regular cycles were less insulin resistant than patients with secondary amenorrhea alternating with regular cycles. Finally, patients with isolated polymenorrhea and those with polymenorrhea alternating with regular cycles had comparable levels of markers of IR with Group D.

Conclusions: Amenorrhea is associated with more pronounced IR in PCOS, and oligomenorrhea portends a less excessive risk for IR than amenorrhea whereas polymenorrhea appears to be even more benign metabolically. Therefore, the type of menstrual cycle abnormality appears to represent a useful tool for identifying a more adverse metabolic profile in PCOS.
\end{abstract}

European Journal of Endocrinology 168 145-152

\section{Introduction}

The polycystic ovary syndrome (PCOS) is a frequent endocrine disorder with considerable heterogeneity in its manifestations $(1,2)$. A substantial proportion of patients with PCOS have insulin resistance (IR), which results in increased risk for impaired glucose tolerance and type 2 diabetes mellitus (T2DM) (1, 2, 3, 4). Accumulating data suggest a higher incidence of cardiovascular disease (CVD) in this syndrome and IR appears to contribute to this increased risk $(5,6)$.

Given the frequent presence of IR in patients with PCOS and its association with adverse consequences (T2DM and CVD), it is important to identify patients with PCOS who are insulin resistant. Obesity is a major risk factor for IR both in the general population and in patients with PCOS $(2,7)$. However, IR can be present even in normal weight patients with PCOS (8). Oligo- and anovulation are pivotal characteristics of PCOS and also appear to correlate with the presence of IR $(1,2)$. However, assessment of ovulation requires laboratory investigations and is costly. On the other hand, menstrual cycle irregularity is a relatively accurate surrogate of ovulation and is easily obtained from the medical history $(1,9)$. Therefore, menstrual cycle pattern might serve as a marker of IR in patients with PCOS, as IR can induce oligo- or anovulation and thus menstrual cycle irregularity by exacerbating hyperandrogenemia and by disrupting follicular growth (2). Nevertheless, very few small studies evaluated the association between menstruation abnormalities and the endocrine and metabolic characteristics in PCOS $(10,11,12,13)$.

We aimed to assess the relationship between menstrual cycle irregularities and both IR and circulating androgens 
in a large cohort of patients with PCOS. We also aimed to determine whether different types of menstruation abnormalities are associated with more pronounced IR and hyperandrogenemia in this population.

\section{Materials and methods}

\section{Patients}

We studied 1285 women with PCOS (mean age 24.3 \pm 5.8 years, mean BMI $26.7 \pm 6.9 \mathrm{~kg} / \mathrm{m}^{2}$ ) who were outpatients at the Gynecological Endocrinology Infirmary of the Second Department of Obstetrics and Gynecology, Aristotle University of Thessaloniki. Diagnosis of PCOS was based on the revised criteria of Rotterdam (14). None of the women studied had galactorrhea or any endocrine or systemic disease that could possibly affect reproductive physiology. A Synachten test was performed with tetracosactide (Synachten $0.25 \mathrm{mg} / 1 \mathrm{ml}$; Novartis Pharma) in women with a basal $17 \alpha$-hydroxyprogesterone (17 $\alpha$-OHP) plasma level $>1.5 \mathrm{ng} / \mathrm{ml}$ to exclude congenital adrenal hyperplasia. No woman reported use of any medication that could interfere with the normal function of the hypothalamicpituitary-gonadal axis during the last semester.

Informed consent was obtained from all women, and the study was approved by the Institutional Review Board; the study met the requirements of the 1975 Helsinki guidelines.

\section{Study protocol}

In all women, weight, height, waist circumference (W), and hip circumference $(\mathrm{H})$ were measured. Body weight was measured in light clothing using an analog scale and height was measured barefoot using a stadiometer. BMI was calculated by dividing weight (kilogram) by height squared (meter) to assess obesity. The $\mathrm{W}$ was obtained as the smallest circumference at the level of the umbilicus and the $\mathrm{H}$ was measured at the level of the widest diameter around the buttocks. The $\mathrm{W}$-to-H ratio (WHR) was calculated by dividing $\mathrm{W}$ by $\mathrm{H}$.

Baseline blood samples were collected between days 3 and 7 of the menstrual cycle in women with regular menstrual cycles and after a spontaneous bleeding episode in women with menstrual cycle abnormalities, after an overnight fast. The circulating levels of FSH, LH, prolactin (PRL), total testosterone, $\Delta_{4}$-androstenedione, DHEAS, $17 \alpha-\mathrm{OHP}$, sex hormone-binding globulin (SHBG), glucose, and insulin were measured. Immediately after baseline blood sampling, an oral glucose tolerance test (OGTT) was performed; $75 \mathrm{~g}$ glucose were administered orally and serum glucose levels were determined after 30, 60, 90, and $120 \mathrm{~min}$. On the same day, transvaginal ultrasonography was performed and the volume of each ovary was determined, as well as the number of small follicles (measuring 2-9 $\mathrm{mm}$ in diameter) in each ovary.
Women with PCOS were divided according to the menstrual cycle pattern into i) women with a single cycle irregularity (i.e. with primary amenorrhea $(n=4)$, secondary amenorrhea $(n=37)$, oligomenorrhea $(n=95)$, or polymenorrhea $(n=9)$; Group A); ii) women with multiple cycle irregularities (i.e. with secondary amenorrhea alternating with oligomenorrhea $(n=82)$ or polymenorrhea $(n=23)$ and women with oligomenorrhea alternating with polymenorrhea $(n=147)$; Group B); iii) women with regular menstrual cycles alternating with a single cycle irregularity (i.e. with secondary amenorrhea $(n=106)$, oligomenorrhea $(n=598)$, or polymenorrhea $(n=53)$; Group $C)$; and iv) women with regular menstrual cycles $(n=131$; Group D) (Fig. 1). Menstrual cycle data were obtained from a diary that women were keeping for 1 year before enrollment to the study. For the previous years, menstrual cycle data were provided from women from memory. Primary amenorrhea was defined as the absence of menstruation by the age of 16 years. Secondary amenorrhea was defined as absence of vaginal bleeding for at least 6 months after a period of established menstruation. Oligomenorrhea was defined as cycle length $>35$ days or $<8$ cycles/year. Polymenorrhea was defined as cycle length $\leq 21$ days. Regular menstrual cycles were defined as cycle length $28 \pm 4$ days (15).

\section{Methods}

Serum FSH, LH, PRL, androgen, 17 $\alpha-\mathrm{OHP}$, SHBG, glucose, insulin, TSH, and free thyroxine $\left(\mathrm{FT}_{4}\right)$ were measured as described previously (16). Free androgen index (FAI) was determined as follows: $\mathrm{FAI}=$ testosterone

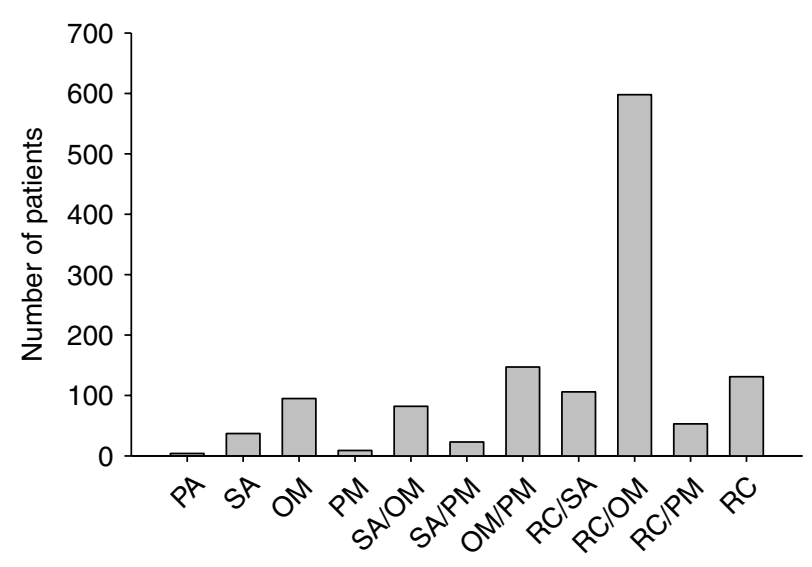

Figure 1 Distribution of menstrual cycle pattern in the study population. PA, primary amenorrhea; SA, secondary amenorrhea; OM, oligomenorrhea; PM, polymenorrhea; SA/OM, secondary amenorrhea alternating with oligomenorrhea; SA/PM, secondary amenorrhea alternating with polymenorrhea; OM/PM, oligomenorrhea alternating with polymenorrhea; $\mathrm{RC}$, regular cycles; $\mathrm{RC} / \mathrm{SA}$, regular cycles alternating with secondary amenorrhea; $\mathrm{RC} / \mathrm{OM}$, regular cycles alternating with oligomenorrhea; $\mathrm{RC} / \mathrm{PM}$, regular cycles alternating with polymenorrhea. 
$(\mathrm{nmol} / \mathrm{l}) \times 100 /$ SHBG $(\mathrm{nmol} / \mathrm{l})(17)$. The homeostasis model assessment of IR (HOMA-IR) index was calculated as follows: HOMA-IR $=$ fasting insulin $(\mu \mathrm{IU} / \mathrm{ml}) \times$ fasting glucose $(\mathrm{mg} / \mathrm{dl}) / 405$ (18). The quantitative insulin sensitivity check index (QUICKI) was calculated according to the following formula: QUICKI $=1 /(\log$ Insulin $(\mu \mathrm{IU} / \mathrm{ml})+\log$ Glucose $(\mathrm{mg} / \mathrm{dl}))(19)$.

\section{Transvaginal ultrasonography}

Transvaginal ultrasound scans of the ovaries were performed by an experienced sonographer in women who participated in the study. Ovarian volume was calculated by the formula: $V=(\pi / 6) \times D_{\text {length }} \times D_{\text {width }} \times$ $D_{\text {thickness, }}$, where $D$ is dimension. The presence of polycystic ovaries was diagnosed by the presence of 12 or more follicles in each ovary measuring $2-9 \mathrm{~mm}$ in diameter and/or increased ovarian volume $\left(>10 \mathrm{~cm}^{3}\right)$.

\section{Statistical analysis}

Data analysis was performed with the statistical package SPSS (version 17.0; SPSS, Inc., Chicago, IL, USA). Data are reported as mean \pm s.D. Differences between groups were assessed using one-way ANOVA with the HolmSidak method for multiple comparison testing. Correction for age, BMI, and W was carried out using analysis of covariance in comparisons among groups with differences in these parameters. Multiple regression analysis was performed to identify independent predictors of IR (assessed with the HOMA-IR) including in the analysis the BMI, the WHR, the menstrual pattern and circulating androgens (assessed with the FAI). In all cases, a $P$ value $<0.05$ was considered significant.

\section{Results}

Characteristics of women with primary amenorrhea are shown in Table 1 . These women $(n=4)$ did not differ in circulating androgens from Group D $(n=131)$ except for FAI, which was higher in women with primary amenorrhea $(P=0.026)$. In addition, women with primary amenorrhea were more insulin resistant than Group D.

Characteristics of women with isolated secondary amenorrhea $(n=37)$ are shown in Table 1 . These women did not differ in circulating androgens from Group D except for FAI and serum DHEAS levels, which were higher in women with isolated secondary amenorrhea $(P<0.001$ and $P=0.006$ respectively). In addition, women with isolated secondary amenorrhea were more insulin resistant than Group D. Characteristics of women with secondary amenorrhea alternating with regular menstrual cycles $(n=106)$ are shown in Table 2 . These women had higher circulating androgens and were more insulin resistant than Group D.
Characteristics of women with isolated oligomenorrhea $(n=95)$ are shown in Table 1 . These women did not differ in circulating androgens from Group D except for FAI, which was higher in women with isolated oligomenorrhea $(P=0.009)$. In addition, women with isolated oligomenorrhea were more insulin resistant than Group D. In contrast, women with oligomenorrhea alternating with regular menstrual cycles, secondary amenorrhea, or polymenorrhea had comparable levels of circulating androgens and markers of IR with Group D (Tables 2 and 3). On the other hand, women with oligomenorrhea alternating with regular menstrual cycles had lower circulating androgens and were less insulin resistant than women with secondary amenorrhea alternating with regular menstrual cycles (Table 2).

Characteristics of women with isolated polymenorrhea $(n=9)$ are shown in Table 1 . These women did not differ in circulating androgens or markers of IR from Group D. Similarly, women with polymenorrhea alternating with regular menstrual cycles had comparable levels of circulating androgens and markers of IR with Group D (Table 2). In contrast, women with polymenorrhea alternating with regular menstrual cycles had lower circulating androgens than women with secondary amenorrhea alternating with regular menstrual cycles. In addition, women with polymenorrhea alternating with regular menstrual cycles were less insulin resistant than women with regular menstrual cycles alternating with either secondary amenorrhea or oligomenorrhea. Finally, women with polymenorrhea alternating with oligomenorrhea had lower FAI than women with secondary amenorrhea alternating with either polymenorrhea or oligomenorrhea $(P=0.003$ and $P=0.006$ respectively) whereas markers of IR were comparable (Table 3).

In multiple regression analysis, independent predictors of HOMA-IR were the BMI $(P<0.001)$, the FAI $(P=0.028)$, and the menstrual pattern $(P<0.001)$. In post hoc analysis, among the different types of menstrual pattern, only primary amenorrhea, isolated secondary amenorrhea, isolated oligomenorrhea, and secondary amenorrhea alternating with oligomenorrhea were independent predictors of HOMA-IR compared with Group D $(P<0.001, \quad P=0.004$, $P=0.004$, and $P=0.037$ respectively).

\section{Discussion}

This is the largest study that evaluated the association between menstrual cycle pattern and IR/androgen levels in patients with PCOS. We report that patients with isolated primary amenorrhea, secondary amenorrhea, or oligomenorrhea and patients with secondary amenorrhea alternating with regular menstrual cycles had more pronounced IR than Group D. In contrast, patients with oligomenorrhea alternating with 


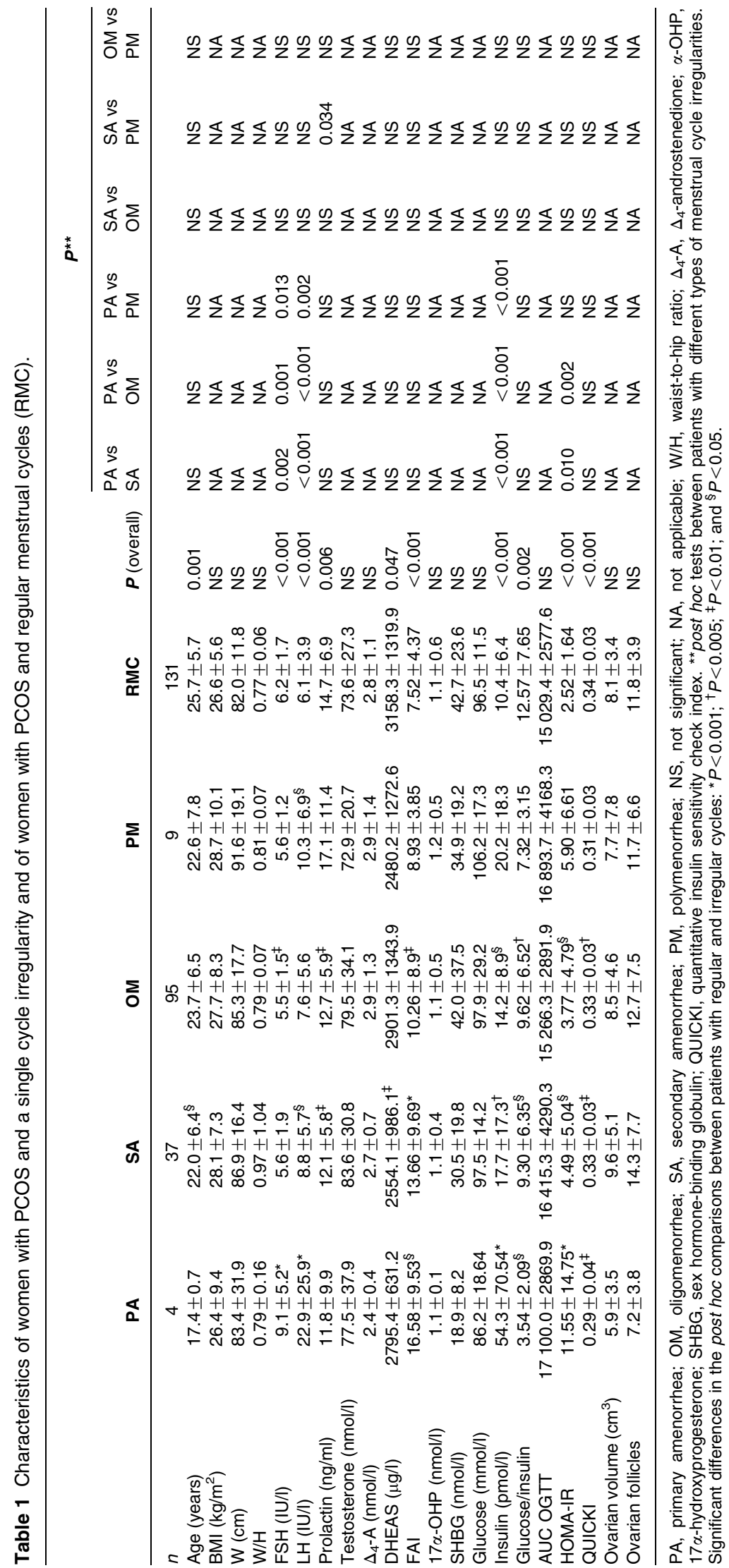




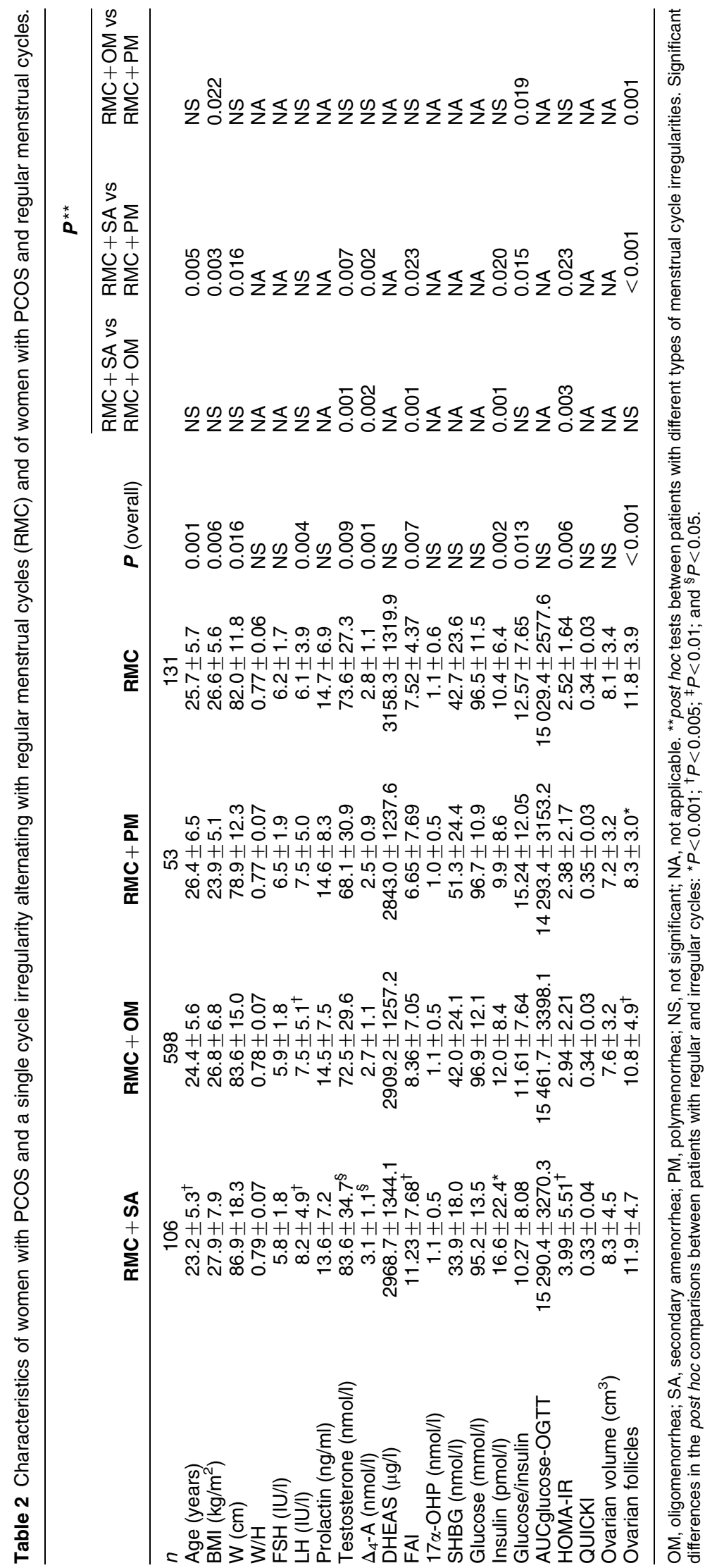




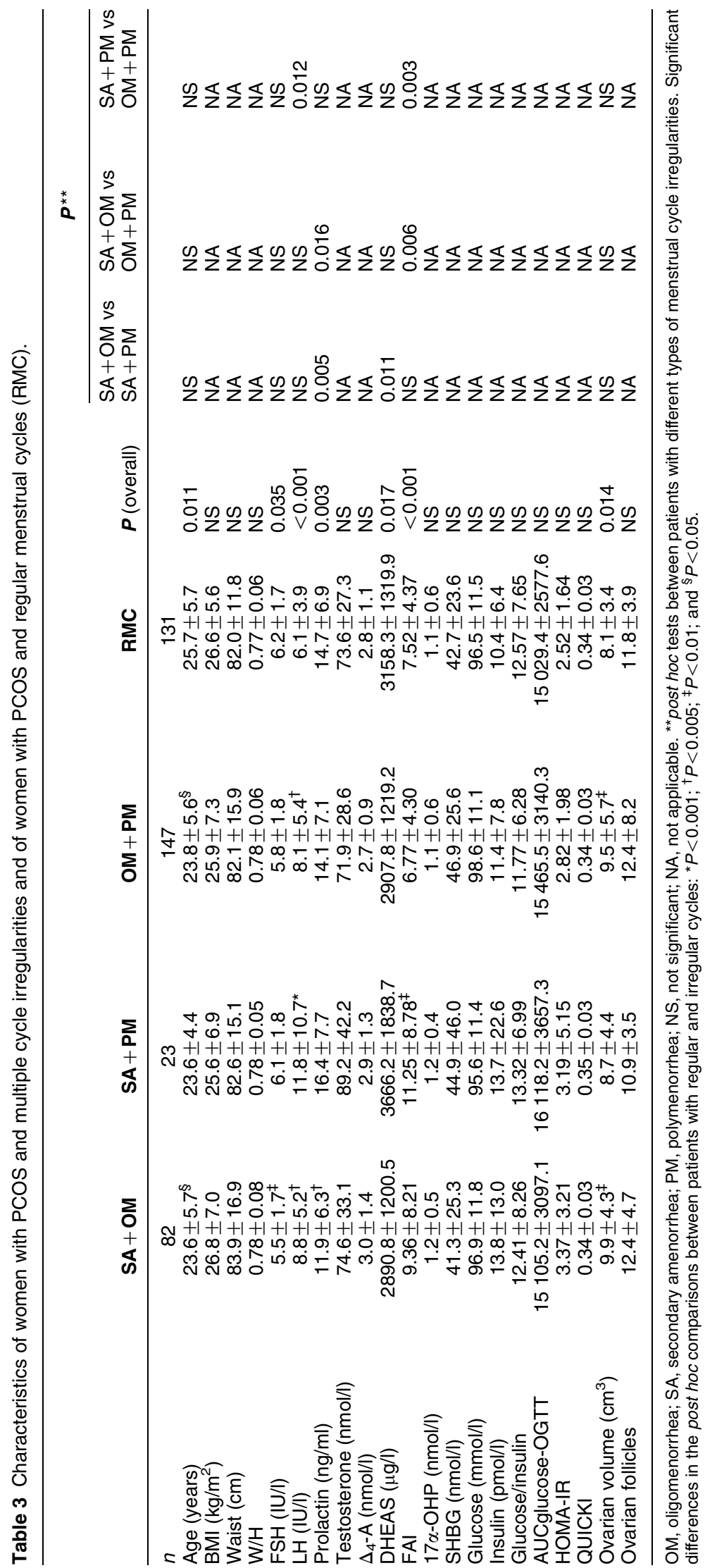


secondary amenorrhea or with regular menstrual cycles did not differ in markers of IR from Group D. Two previous smaller studies $(n=72$ and $n=418$ respectively) reported that patients with PCOS and oligomenorrhea or amenorrhea had more severe IR than patients with PCOS and regular cycles $(10,11)$. However, the latter studies did not differentiate between patients with oligomenorrhea and amenorrhea $(10,11)$. In contrast, in two more recent small studies ( $n=184$ and $n=118$ respectively) that analyzed patients with amenorrhea separately from patients with oligomenorrhea, only the former had more pronounced IR than patients with regular menses $(12,13)$. Markers of IR did not differ between patients with oligomenorrhea and patients with regular menses $(12,13)$. We also observed that women with regular menstrual cycles alternating with secondary amenorrhea were more insulin resistant than women with regular menstrual cycles alternating with oligomenorrhea. There are no studies that compared markers of IR between different types of menstrual abnormalities in patients with PCOS. Overall, our findings suggest that amenorrhea is a useful marker of IR in patients with PCOS whereas oligomenorrhea does not imply more severe IR. This association is possibly explained by the relationship between IR and anovulation. Indeed, most patients with PCOS and amenorrhea are anovulatory and IR contributes to the pathogenesis of anovulation in PCOS by aggravating hyperandrogenemia and by inducing ovarian follicular arrest $(1,2)$. Therefore, the more severe IR might partly explain the presence of amenorrhea in these patients. On the other hand, patients with oligomenorrhea might have less perturbed ovulation partly because they have less severe IR. Interestingly, a recent study showed that the hormonal/ metabolic profile is comparable in women with PCOS despite the time of menstrual irregularities occurrence (20). This observation and our findings in this study suggest that the type of menstrual irregularity might be more important than the duration of PCOS in determining the metabolic profile of these women.

This is the first study that assessed the association between polymenorrhea and the metabolic and endocrine characteristics of patients with PCOS. We observed that patients with polymenorrhea alone or in combination with other cycle abnormalities or alternating with regular menstrual cycles did not differ in markers of IR from Group D. Moreover, patients with regular menstrual cycles alternating with polymenorrhea were less insulin resistant than patients with regular menstrual cycles alternating with either secondary amenorrhea or oligomenorrhea. These results suggest that polymenorrhea is associated with milder impairment in glucose metabolism than amenorrhea and oligomenorrhea. Indeed, in the general population, women with oligomenorrhea appear to have increased risk for T2DM whereas those with polymenorrhea have an incidence of T2DM similar to women with regular menses (20). However, when clinical signs of hyperandrogenemia (hirsutism and/or acne) were present in women with polymenorrhea, the risk of T2DM increased (21). Clearly, more studies are required to confirm or refute the relationship between polymenorrhea and IR/T2DM and to clarify the mechanism(s) underpinning this association.

Differences in circulating androgens paralleled differences in markers of IR. Indeed, patients with primary or secondary amenorrhea or oligomenorrhea had more severe hyperandrogenemia than Group D whereas patients with polymenorrhea had a similar degree of hyperandrogenemia with the latter. A previous study reported higher androgen levels in patients with either amenorrhea or oligomenorrhea than in those with normal cycles (13) whereas another did not detect any differences in androgen levels between the three groups, possibly because of limited statistical power (12). Therefore, it appears that the severity of menstrual abnormality also reflects the degree of hyperandrogenemia. Indeed, ovarian hyperandrogenism is associated with anovulation in PCOS by inducing ovarian follicular arrest (2, 22). Accordingly, the type of menstrual cycle irregularity might also represent an inexpensive and easily determined marker of hyperandrogenemia in patients with PCOS.

In conclusion, amenorrhea is associated with more pronounced IR and hyperandrogenemia in patients with PCOS. Oligomenorrhea portends a less excessive risk for these abnormalities than amenorrhea whereas polymenorrhea appears to be even more benign metabolically. Therefore, the type of menstrual cycle abnormality might represent a useful tool for identifying a more severe metabolic profile in PCOS.

\section{Declaration of interest}

The authors declare that there is no conflict of interest that could be perceived as prejudicing the impartiality of the research reported.

\section{Funding}

This research did not receive any specific grant from any funding agency in the public, commercial or not-for-profit sector.

\section{References}

1 Norman RJ, Dewailly D, Legro RS \& Hickey TE. Polycystic ovary syndrome. Lancet $2007 \mathbf{3 7 0}$ 685-697. (doi:10.1016/S01406736(07)61345-2)

2 Goodarzi MO, Dumesic DA, Chazenbalk G \& Azziz R. Polycystic ovary syndrome: etiology, pathogenesis and diagnosis. Nature Reviews. Endocrinology 20117 219-231. (doi:10.1038/nrendo. 2010.217)

3 Boudreaux MY, Talbott EO, Kip KE, Brooks MM \& Witchel SF. Risk of T2DM and impaired fasting glucose among PCOS subjects: results of an 8-year follow-up. Current Diabetes Reports 20066 77-83. (doi:10.1007/s11892-006-0056-1)

4 Moran LJ, Misso ML, Wild RA \& Norman RJ. Impaired glucose tolerance, type 2 diabetes and metabolic syndrome in polycystic 
ovary syndrome: a systematic review and meta-analysis. Human Reproduction Update 201016 347-363. (doi:10.1093/humupd/ dmq001)

5 Shaw LJ, Bairey Merz CN, Azziz R, Stanczyk FZ, Sopko G, Braunstein GD, Kelsey SF, Kip KE, Cooper-Dehoff RM, Johnson BD et al. Postmenopausal women with a history of irregular menses and elevated androgen measurements at high risk for worsening cardiovascular event-free survival: results from the National Institutes of Health - National Heart, Lung, and Blood Institute sponsored Women's Ischemia Syndrome Evaluation. Journal of Clinical Endocrinology and Metabolism 2008 93 1276-1284. (doi:10.1210/jc.2007-0425)

6 Wild RA, Carmina E, Diamanti-Kandarakis E, Dokras A, EscobarMorreale HF, Futterweit W, Lobo R, Norman RJ, Talbott E \& Dumesic DA. Assessment of cardiovascular risk and prevention of cardiovascular disease in women with the polycystic ovary syndrome: a consensus statement by the Androgen Excess and Polycystic Ovary Syndrome (AE-PCOS) Society. Journal of Clinical Endocrinology and Metabolism 201095 2038-2049. (doi:10. 1210/jc.2009-2724)

7 Iozzo P. Viewpoints on the way to the consensus session: where does insulin resistance start? The adipose tissue. Diabetes Care 200932 (Suppl 2) S168-S173. (doi:10.2337/dc09-S304)

8 Dunaif A, Segal KR, Futterweit W \& Dobrjansky A. Profound peripheral insulin resistance, independent of obesity, in polycystic ovary syndrome. Diabetes 198938 1165-1174. (doi:10.2337/ diabetes.38.9.1165)

9 Azziz R, Waggoner WT, Ochoa T, Knochenhauer ES \& Boots LR. Idiopathic hirsutism: an uncommon cause of hirsutism in Alabama. Fertility and Sterility 199870 274-278. (doi:10. 1016/S0015-0282(98)00141-1)

10 Robinson S, Kiddy D, Gelding SV, Willis D, Niththyananthan R, Bush A, Johnston DG \& Franks S. The relationship of insulin insensitivity to menstrual pattern in women with hyperandrogenism and polycystic ovaries. Clinical Endocrinology 1993 39 351-355. (doi:10.1111/j.1365-2265.1993.tb02376.x)

11 Welt CK, Gudmundsson JA, Arason G, Adams J, Palsdottir H, Gudlaugsdottir G, Ingadottir G \& Crowley WF. Characterizing discrete subsets of polycystic ovary syndrome as defined by the Rotterdam criteria: the impact of weight on phenotype and metabolic features. Journal of Clinical Endocrinology and Metabolism 200691 4842-4848. (doi:10.1210/jc.2006-1327)

12 Cupisti S, Kajaia N, Dittrich R, Duezenli HW, Beckmann M \& Mueller A. Body mass index and ovarian function are associated with endocrine and metabolic abnormalities in women with hyperandrogenic syndrome. European Journal of Endocrinology 2008158 711-719. (doi:10.1530/EJE-07-0515)

13 Strowitzki T, Capp E \& von Eye Corleta H. The degree of cycle irregularity correlates with the grade of endocrine and metabolic disorders in PCOS patients. European Journal of Obstetrics, Gynecology, and Reproductive Biology $2010 \quad 149$ 178-181. (doi:10.1016/j.ejogrb.2009.12.024)

14 Rotterdam ESHRE/ASRM-Sponsored PCOS Consensus Workshop Group. Revised 2003 consensus on diagnostic criteria and longterm health risks related to polycystic ovary syndrome. Fertility and Sterility 200481 19-25. (doi:10.1016/j.fertnstert.2003. 10.004)

15 De Cherney AH, Nathan L, Murphy Goodwin T \& Laufer N. Current Diagnosis and Treatment Obstetrics \& Gynecology, 10th edn, Columbus: The McGraw-Hill Companies, 2006.

16 Piouka A, Farmakiotis D, Katsikis I, Macut D, Gerou S \& Panidis D. Anti-Müllerian hormone levels reflect severity of PCOS but are negatively influenced by obesity: relationship with increased luteinizing hormone levels. American Journal of Physiology. Endocrinology and Metabolism 2009296 E238-E243. (doi:10. 1152/ajpendo.90684.2008)

17 Carter GD, Holland SM, Alaghband-Zadeh J, Rayman G, DorringtonWard P \& Wise PH. Investigation of hirsutism: testosterone is not enough. Annals of Clinical Biochemistry 198320 262-263.

18 Matthews DR, Hosker JP, Rudenski AS, Naylor BA, Treacher DF \& Turner RC. Homeostasis model assessment: insulin resistance and $\beta$-cell function from fasting plasma glucose and insulin concentrations in man. Diabetologia 198528 412-419. (doi:10.1007/ BF00280883)

19 Katz A, Nambi SS, Mather K, Baron AD, Follmann DA, Sullivan G \& Quon MJ. Quantitative insulin sensitivity check index: a simple, accurate method for assessing insulin sensitivity in humans. Journal of Clinical Endocrinology and Metabolism 2000 85 2402-2410. (doi:10.1210/jc.85.7.2402)

20 Livadas S, Christou M, Economou F, Karachalios A, Xyrafis X, Boutzios G, Zerva A, Tantalaki E, Palimeri S \& DiamantiKandarakis E. Menstrual irregularities in PCOS. Does it matter when it starts? Experimental and Clinical Endocrinology and Diabetes 2011119 334-337. (doi:10.1055/s-0030-1269882)

21 Solomon CG, Hu FB, Dunaif A, Rich-Edwards J, Willett WC, Hunter DJ, Colditz GA, Speizer FE \& Manson JE. Long or highly irregular menstrual cycles as a marker for risk of type 2 diabetes mellitus. Journal of the American Medical Association 2001286 2421-2426. (doi:10.1001/jama.286.19.2421)

22 Agarwal SK, Judd HL \& Magoffin DA. A mechanism for the suppression of estrogen production in polycystic ovary syndrome. Journal of Clinical Endocrinology and Metabolism 1996 81 3686-3691. (doi:10.1210/jc.81.10.3686)

Received 30 July 2012

Revised version received 8 October 2012

Accepted 29 October 2012 